\title{
Primary hydatid cyst of soleus muscle : A case report
}

Nahar $\mathrm{MN}^{1}$, Iqbal $\mathrm{H}^{2}$, Yesmin $\mathrm{MS}^{3}$, Arafat $\mathrm{SM}^{4}$

\begin{abstract}
Muscle hydatidosis is rare, accounting only for 3-5\% of all cases. We present an unusual case of a primary hydatid cyst found in the soleus muscle of a 42-year-old woman, presenting as an enlarging soft-tissue mass for 6 months associated with pain. The hydatid cyst was confined into muscle, without affecting the bone. En block surgical excision of the cyst was undertaken. Histopathologic findings confirmed the diagnosis. No recurrence occurred during the follow-up period of 8 months. Skeletal muscle echinococcosis should be considered in the differential diagnosis of limb masses, especially in endemic countries. A meticulous history taking and MR imaging is essential, while pericystectomy is an effective method of treatment.
\end{abstract}

Keywords: Hydatid cyst, skeletal muscle hydatosis, echinococcosis

\section{Introduction}

Hydatid disease, which is a zoonotic infection caused by larval forms (metacestodes) of tapeworms of the genus echinococcus found in the small intestine of carnivores, still remains an important health problem in endemic regions. ${ }^{1,2}$ Two of the four recognized species of echinococcus, E. granulosus and E. multilocularis, cause cystic echinococcosis (CE) and alveolar echinococcosis (AE) in humans, respectively. The eggs of these tapeworms excreted by carnivores may infect humans as natural intermediate host. $^{2}$ The disease results from the development of the larval or hydatid form in the body. Hydatid cysts, which generally involve the liver and the lungs, are uncommonly found in muscles; even in endemic zones. ${ }^{2}$ Exclusive involvement of the muscles is extremely uncommon, because implantation at this site would

1. *Dr Most. Naznin Nahar, Associcte Professor of Pathology Popular Medical College, Dhaka, Bangladesh

2. Dr Hasina Iqbal, Assistant Professor of Microbiology Popular Medical College, Dhaka, Bangladesh

3. Dr Most. Shaila Yesmin, Assistant Professor of Pathology Dhaka Community Medical College, Dhaka, Bangladesh

4. Dr SM Arafat, Associate Professor of Medicine BSMMU, Dhaka, Bangladesh

${ }^{*}$ For correspondence require passage through the filters of the liver and lung. We describe a patient with pain and a mass in the soleus muscle for 6 months, who was found to have hydatid disease.

\section{Case report}

A 42-year-old female patient presented to a surgeon in Dhaka, Bangladesh with the complaints of swelling of the right calf region associated with pain for six months. The swelling was gradually increasing in size. The pain was aggravated on walking. On examination, no associated tenderness was found. The radius of the swelling was $5 \mathrm{~cm}$. The cyst did not disappear or reduced in size on flexion of the knee joint. Illumination test was negative. Fluctuation was present. Skin over the swelling was normal. X-ray of knee joint did not reveal any abnormality. Ultrasonography (USG) suggested Backer's cyst. Routine blood tests ( $\mathrm{Hb} \%$, total and differential leukocyte counts, serum creatinine and urea level, and fasting blood glucose) were found within normal limit. Chest X-ray (PA view) and electrocardiogram were also found within normal limit. She was planned for excision of the cyst.

During the operation a white cystic mass was successfully extirpated. Pathological examination revealed two ivory white deflated cysts measuring $4 \mathrm{~cm}$ and $2 \mathrm{~cm}$ respectively with wall thickness of $0.1 \mathrm{~cm}$ on gross examination (Figure-1). Microscopic examination revealed acellular hyalinized laminated membranes of hydatid cyst wall with attached protoscolices (Figure-2).

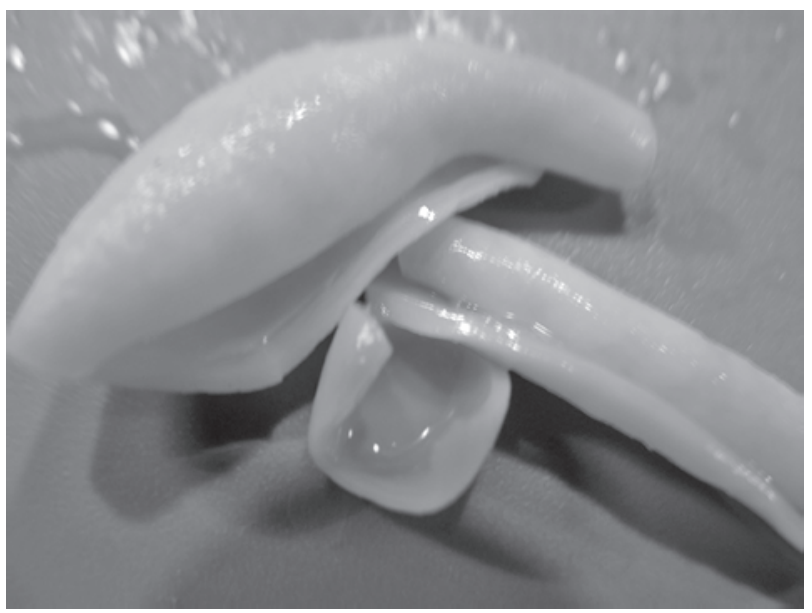

Figure-1: Ivory white cyst wall of hydatid cyst 


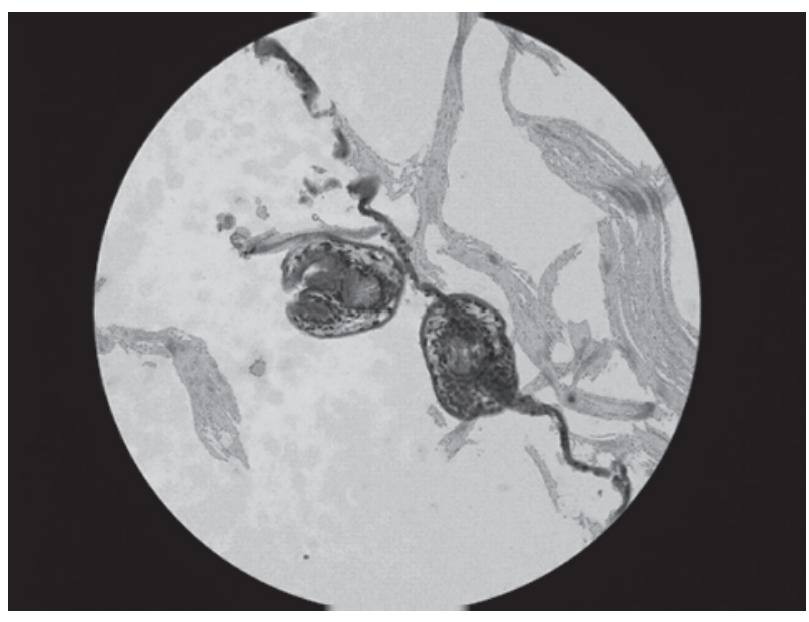

Figure-2: Acellular laminated membranes of protoscolices (HङE stain) of hydatid daughter cyst

The patient was discharged from the hospital in her third postoperative day uneventfully and post-operatively she underwent Albendazole treatment. We performed echocardiography, computed tomography of thorax and abdomen to exclude other possible localizations of hydatidosis. According to this work-up, we concluded this case as a primary skeletal muscle hydatidosis.

\section{Discussion}

Muscle hydatidosis is uncommon, accounting only for $3-5 \%$ of all cases 3,4 and is usually secondary to hepatic or pulmonary disease. Most of the cases reported quadriceps, ${ }^{3,5}$ gluteus, ${ }^{6}$ trapezius, ${ }^{7}$ psoas, ${ }^{8}$ erector spinae, ${ }^{9}$ biceps femoris ${ }^{3,10}$ adductor brevis, ${ }^{11}$ infraspinatus, ${ }^{3}$ biceps brachii, ${ }^{6,12}$ and triceps brachii ${ }^{3}$ as the muscles of involvement. Muscle hydatidosis involving soleus muscle in the popliteal fossa is very rare. Till now no case was reported. 3,13

Usually, intramuscular hydatid cysts are secondary, resulting either from the spread of cysts or viable larval tissue after spontaneous or trauma-induced cyst rupture or after operations for hydatidosis in distant regions. ${ }^{1}$

Several factors would explain the exceptional nature of muscle localizations of hydatid cysts - efficacy of the hepatic and pulmonary barriers, unfavorable muscle environment for the growth of hydatid larvae due to high lactic acid content and muscle's contractility which hinders intramuscular growth of cysts. ${ }^{4}$ The predominant localization in the proximal muscles of the lower limbs could be explained by the volume of the muscle mass and its rich blood supply.

It is important to establish the diagnosis preoperatively in order to limit the risk of anaphylactic shock or dissemination of viable protoscolices in the event of puncture or accidental opening of the cyst during resection. Chest radiography, USG, computed tomography (CT), and magnetic resonance (MR) imaging are all can be used to depict hydatid cyst. However, the imaging method to be used depends on the organ involved and the growth stage of the cyst. Intramuscular hydatid disease can cause a variety of diagnostic problems, especially in the absence of typical radiologic findings. Diagnostic problem happened in our case also, where the USG wrongly suggested the presence of Backer's cyst. Nevertheless, USG is the diagnostic tool of choice for the initial work-up and is particularly useful for detection of the floating membranes, daughter cysts, and hydatid sand in purely cystic lesions. ${ }^{14}$ It is also used for staging and classification of E. granulosus infection. ${ }^{15} \mathrm{CT}$ is best for detecting cyst wall calcification and revealing the internal cystic structure posterior to calcification, and is the modality of choice in peritoneal seeding. ${ }^{16}$ Both CT and MR imaging can detect cyst wall defects as well as the complications such as rupture and infection of cysts. ${ }^{16}$

Immunodiagnosis can also play an important complementary role for primary diagnosis and also for follow-up of patients after surgical or pharmacological treatment. Detection of circulating E. granulosus antigens in sera is less sensitive than antibody detection, which remains the method of choice. Enzyme-linked immunosorbent assay (ELISA), the indirect immunofluorescence antibody test, immunoelectrophoresis (IEP), and immunoblotting (IB) are the routine laboratory tests used for serological diagnosis. The sensitivity and specificity of these tests vary according to the nature and source of the antigens used. In different studies when $\mathrm{AgB}$ (one of the two antigens used for serology) was used as antigen, both ELISA and immunoblotting have shown specificity up to $100 \%$ and sensitivity up to $89 \%$ and $92 \%$, respectively. ${ }^{17}$

Complete surgical treatment offers best hope for permanent cure and should include excision of the primary lesion, the daughter cysts and the communicating fistulas as a whole specimen. ${ }^{1}$ However, extension of cyst in different muscle layers through communicating fistulas involving different muscle groups may cause difficulty in complete excision and also spillage of daughter cysts resulting in recurrence. Concomitant drug treatment with antihelminthics such as Albendazole or Praziquantel reduces the risk of secondary echinococcosis and recurrence. ${ }^{1,18}$ Prior injection of a scolicidal agent into the unopened cyst and mopping the operative field with sponges soaked in a scolicidal agent are the two most commonly employed measures. Hypertonic saline (15-20\%), cetrimide $(0.5 \%)$, chlorhexidine, hydrogen peroxide, and ethyl alcohol (70-95\%) are some of the compounds used as scolicidal. ${ }^{1}$ 
We used Savlon (setrimide) as scolicidal agent because of its ready availability in the operation theatre. Savlon has also been proved to be the least concentration dependent and most effective scolicidal agent. ${ }^{19}$

Muscular hydatidosis is very rare and can cause difficulty in diagnosis, especially in the absence of typical radiological findings. The possibility of hydatid disease should always be kept in mind in the differential diagnosis of a cystic mass in the muscle, especially in endemic areas to avoid fine-needle biopsy and the consequences of spillage of cyst contents.

\section{References}

1. Guidelines for treatment of cystic and alveolar echinococcosis in humans. WHO Informal Working Group on Echinococcosis. Bull World Health Organ. 1996; 74:231-242.

2. Eckert J, Deplazes P. Biological, epidemiological, and clinical aspects of echinococcosis, a zoonosis of increasing concern. Clin Microbiol Rev. 2004;17:107-135.

3. Gougoulias NE, Varitimidis SE, Bargiotas KA, Dovas TN, Karydakis G, Dailiana ZH. Skeletal muscle hydatid cysts presenting as soft tissue masses. Hippokratia. 2010;14:126-130.

4. García-Alvarez F, Torcal J, Salinas JC, Navarro A, García-Alvarez I, Navarro-Zorraquino $\mathrm{M}$ et al. Musculoskeletal hydatid disease: A report of 13 cases. Acta Orthop Scand. 2002;73:227-231.

5. Ozkoç G, Akpinar S, Hersekli MA, Ozalay M, Tandoðan R. Primary hydatid disease of the quadriceps muscle: A rare localization. Arch Orthop Trauma Surg. 2003; 123:314-166.

6. Ates M, Karakaplan M. Hydatid cyst in the biceps and gluteus muscles: Case report. Surg Infect (Larchmt)2007;8:475-478.

7. Calò PG, Tatti A, Tuveri M, Farris S, Nicolosi A. Hydatid cyst of trapezius muscle: An unusual localisation. Report of a case and review of the literature. Chir Ital. 2007;59:873-876.
8. Bilanoviæ D, Zdravkoviæ $\mathrm{D}$, Randjeloviæ $\mathrm{T}$, Toskoviæ B, Gaciæ J. Lesion of the femoral nerve caused by a hydatid cyst of the right psoas muscle. Srp Arh Celok Lek. 2010;138:502-505.

9. Atalay F, Orug T, Arda K, Tosun O, Atalay F. An unusual case of hydatid disease located in the erector spinae muscle. JBR-BTR 2003;86:329-331

10. Hamdi MF, Touati B, Abid A. Primary hydatid cyst of the biceps femoris. Musculoskelet Surg. 2010;94:59-61.

11. Acar A, Rodop O, Yenilmez E, Baylan O, Oncül O. Case report: Primary localization of a hydatid cyst in the adductor brevis muscle. Turkiye Parazitol Derg. 2009; 33:174-176.

12. Karapinar H, Yaðdi S, Durmuº K, Sener M. Primary hydatid disease of the biceps brachii. J Shoulder Elbow Surg. 2008;17:6-8.

13. Seijas R, Catalán-Larracoechea JM, Ares-Rodríguez O, Joshi N, de la Fuente JP, Pérez-Domínguez M. Primary hydatid cyst of skeletal muscle affecting the knee: A case report. Arch Orthop Trauma Surg. 2009;129:39-41.

14. Turgut AT, Akhan O, Bhatt S, Dogra VS. Sonographic spectrum of hydatid disease. Ultrasound Q. 2008 ; 24 : 17-29.

15. WHO Informal Working Group. International classification of ultrasound images in cystic echinococcosis for application in clinical and field epidemiological settings. Acta Trop. 2003; 85:253-261.

16. Pedrosa I, Saíz A, Arrazola J, Ferreirós J, Pedrosa CS. Hydatid disease: Radiologic and pathologic features and complications. Radiographics. 2000;20:795-817.

17. Zhang W, Li J, McManus DP. Concepts in immunology and diagnosis of hydatid disease. Clin Microbiol Rev. 2003;16:18-36.

18. Shams-Ul-Bari, Arif SH, Malik AA, Khaja AR, Dass TA, Naikoo ZA. Role of albendazole in the management of hydatid cyst liver. Saudi J Gastroenterol. 2011; 17:343-347.

19. Besim H, Karayalçin K, Hamamci O, Güngör C, Korkmaz A. Scolicidal agents in hydatid cyst surgery. HPB Surg. 1998;10:347-351 